\title{
S323I polymorphism of the C5L2 gene was not identified in a Chinese population with familial combined hyperlipidemia or with type 2 diabetes
}

\author{
Y.-Y. Zheng ${ }^{1,2}$, X. Xie ${ }^{1,2}$, Y.-T. Ma ${ }^{1,2}$, Y.-N. Yang ${ }^{1,2}$, Z.-Y. Fu ${ }^{1,2}$, X.-M. Li ${ }^{1,2}$, \\ F. Liu' ${ }^{2}$, S.-J. Yang ${ }^{1,2}$, X. Ma ${ }^{1,2}$ and B.-D. Chen ${ }^{2}$ \\ ${ }^{1}$ Department of Cardiology, \\ First Affiliated Hospital of Xinjiang Medical University, \\ Urumqi, P.R. China \\ ${ }^{2}$ Xinjiang Key Laboratory of Cardiovascular Disease Research, \\ Urumqi, P.R. China \\ Corresponding author: Y.-T. Ma \\ E-mail: myt-xj@163.com / myt_xj@sina.com
}

Genet. Mol. Res. 10 (4): 3256-3266 (2011)

Received February 3, 2011

Accepted August 13, 2011

Published December 22, 2011

DOI http://dx.doi.org/10.4238/2011.December.22.4

\begin{abstract}
C5L2, a G protein-coupled receptor, is known to be a functional receptor of acylation-stimulating protein, which is a stimulator of triglyceride synthesis and glucose transport. A novel C5L2 variant (S323I) was identified and its association with familial combined hyperlipidemia $(\mathrm{FCH})$ was recently reported. We looked for this SNP in three Chinese ethnic groups, including Han, Uygur, and Kazakh controls and patients with FCH and type 2 diabetes. One hundred and eighty-two unrelated subjects (77 of Han, 57 of Uygur, and 48 of Kazakh) with FCH were genotyped by direct sequencing, and 852 subjects (342 of Han, 338 of Uygur, 172 of Kazakh) with type 2 diabetes and 200 healthy controls (67 of Han, 72 of Uygur,
\end{abstract}


and 61 of Kazakh) chosen from a cardiovascular risk survey study were genotyped with PCR-RFLP analysis. All 182 subjects with $\mathrm{FCH}, 99.5 \%$ of the type 2 diabetes patients and $100 \%$ of the healthy controls were successfully genotyped. Neither the FCH subjects nor the type 2 diabetes patients were found to have the S323I variant. This variant was also not identified in the healthy controls. We found no evidence to demonstrate that the S323I polymorphism contributes to familial combined hyperlipidemia or type 2 diabetes in the Chinese population.

Key words: Acylation-stimulating protein; Triglyceride synthesis; C5L2; Familial combined hyperlipidemia

\section{INTRODUCTION}

Acylation-stimulating protein (ASP), also known as C3a des-Arg, as a stimulator of either triglyceride synthesis (Faraj et al., 2004; Xiang et al., 1999; Cui et al., 2009a; Paglialunga et al., 2009) or glucose transport (Saleh et al., 2009; de Lind van Wijngaarden et al., 2010) has previously been well documented. C5L2, a G protein-coupled receptor, was demonstrated to be a functional receptor of ASP (MacLaren et al., 2007; Cui, et al. 2009b). Kalant et al. (2005) previously demonstrated that ASP binds to C5L2, initiating a cascade of events that includes phosphorylation, $\beta$-arrestin translocation, and receptor internalization. Activation of C5L2 initiates a signaling pathway that includes protein kinase $\mathrm{C}$ activation and translocation and glucose transporter translocation (Cianflone, 2003; Cianflone et al., 2003; Maslowska et al., 2005). Also the activation of this pathway results in increased glucose transport and fatty acid esterification, leading to a net accumulation of adipose triglyceride stores. Therefore, C5L2 was selected as a candidate gene of hyperlipidemia and diabetes mellitus. Although there are 52 single nucleotide polymorphisms (SNPs) for the human C5L2 gene listed in the National Center for Biotechnology Information SNP database (http://www.ncbi.nlm.nih.gov/SNP), the reported studies of these SNPs as regards cardiovascular disease were quite rare. Recently, Marcil et al. (2006) have identified a novel C5L2 variant (S323I) in a French-Canadian family with familial combined hyperlipidemia $(\mathrm{FCH})$. This SNP was associated with increased plasma levels of triglyceride, cholesterol, low-density lipoprotein (LDL) cholesterol, apolipoprotein B, and ASP. Accordingly, this SNP may be an important genetic basis contributing to $\mathrm{FCH}$ and other cardiovascular diseases. However, to date there have been no data reported regarding other ethnicities. In the present study, we attempted to scan this SNP in the three Chinese ethnic groups including Han, Uygur, and Kazakh with FCH or type 2 diabetes mellitus.

\section{MATERIAL AND METHODS}

\section{Subject selection}

The Cardiovascular Risk Survey (CRS) study, as described previously (Xie et al., 2010b), is a multiple-ethnic, community-based, cross-sectional study designed to investigate 
the prevalence, incidence, and risk factors for cardiovascular diseases and to determine the genetic and environmental contributions to atherosclerosis, CAD and cerebral infarction of Chinese Han, Uygur, and Kazakh populations in Xinjiang of western China from October 2007 to March 2010. We used a stratified sampling method to select a representative sample of the general population of Chinese Han, Uygur, and Kazakh of this area. Seven cities (Urumqi, Kelamayi, Hetian, Zhaosu, Fukang, Tulufan, and Fuhai) were chosen and, based on the government records of registered residence, one participant was randomly selected from each household. In this way, a total of 14,618 participants (5757 Hans, 4767 Uygurs, and 4094 Kazakhs), were randomly selected from 26 villages of these seven cities and invited to participate. This study was approved by the Ethics Committee of the First Affiliated Hospital of Xinjiang Medical University and was conducted according to the standards of the Declaration of Helsinki. Written informed consent was obtained from the participants. From these 14,618 people, we recruited 182 unrelated subjects (77 of Han, 57 of Uygur, and 48 of Kazakh) with FCH for the present study. All of these subjects were examined in the absence of lipid-lowering medication. The FCH subjects were identified according to criteria described previously (Marcil et al., 2006). In addition, we also selected a large sample of 852 subjects ( 342 of Han, 338 of Uygur, 172 of Kazakh) with type 2 diabetes and 200 healthy controls (67 of Han, 72 of Uygur, 61 of Kazakh) from the CRS study. Diabetes mellitus was defined as the presence of an active treatment with insulin or an oral anti-diabetic agent; for patients on dietary treatment, documentation of abnormal fasting blood glucose, or glucose tolerance test based on World Health Organization criteria was required for establishing this diagnosis (Xie et al., 2009). The healthy controls were selected according to criteria described previously (Xie et al., 2010a).

\section{Biochemical analysis}

Serum and plasma collected for measurement were immediately frozen at $-80^{\circ} \mathrm{C}$ until analysis. We measured the serum concentration of total cholesterol, HDL cholesterol, LDL cholesterol, triglyceride, and glucose using equipment for chemical analysis (Dimension AR/AVL Clinical Chemistry System, Newark, NJ, USA) employed by the Clinical Laboratory Department of the First Affiliated Hospital of Xinjiang Medical University as described previously (Xiang et al., 2009; Yang et al., 2010).

\section{Sample DNA extraction}

Blood samples were collected with a standard venipuncture technique and EDTAcontaining tubes. DNA was extracted from peripheral vein blood leukocytes using a whole blood genome extraction kit (Beijing Boiteke Corporation, China).

\section{Genotyping of S323I polymorphism}

For these 182 subjects with FCH, genotyping was confirmed by polymerase chain reaction (PCR)-direct sequencing analysis. As regards the 852 patients with type 2 diabetes and those 200 healthy controls, genotyping was performed by PCR-restriction fragment length polymorphism (RFLP) analysis. The primers were designed according to the pro- 
tocol of Marcil et al. (2006) and their synthesis was performed by Shanghai Biological Engineering Company Limited (Shanghai, China). The reaction system and genotyping methods were used as described previously (Marcil et al., 2006). To ensure the results to be verified, we used sequenced genomic DNAs as positive controls in our assays. After being purified, the amplification products were directly sequenced using the dye termination method on an ABI PRISM 3100-Avant Genetic Analyzer (Applied Biosystems, Foster City, CA, USA) employed by the BGI (Huada Gene Company; http://www.genomics.cn/ navigation/index.action; Beijing, China). Of the genotyped samples, $10 \%$ were duplicated and there was at least one positive and one negative control per 96-well DNA plate in our assays. The accuracy of the genotyping was determined by genotype concordance between duplicate samples. We obtained $100 \%$ concordance between the genotyped duplicate samples for each of the SNPs.

\section{RESULTS}

\section{Characteristics of study participants}

The study cohort consists of 182 subjects with $\mathrm{FCH}, 852$ patients with type 2 diabetes, and 200 healthy controls. The clinical and metabolic characteristics of the study population are shown in Table 1, Table 2 and Table 3.

\section{Genotyping results}

The length of amplification products by PCR was 431 bp across the S323I variant. All these 182 subjects with FCH were successfully genotyped by using the direct sequencing method. Also there were 852 patients with type 2 diabetes and 200 healthy controls successfully genotyped by using the RFLP method. However, the S323I variant was not identified in FCH subjects, in diabetic patients and also in healthy controls (Table 4 and Figure 1).

\section{DISCUSSION}

In the present study, the S323I polymorphism of the C5L2 gene was genotyped in 182 subjects with $\mathrm{FCH}, 852$ patients with type 2 diabetes, and 200 healthy controls. We did not identify this variant in these three ethnic groups (Han, Uygur and Kazakh) of Chinese population.

The C5L2 receptor located on chromosome $19 \mathrm{q} 13$ is the region that was identified to be associated with FCH and the pre-diabetic state by genome-wide scan study (Aouizerat et al., 1999; Huertas-Vazquez et al., 2005).

Therefore, we attempted to identify the variations in the C5L2 gene and to analyze their association with $\mathrm{FCH}$, diabetes, and cardiovascular disease. In a previous study, Marcil et al. (2006) identified a novel variant in the C5L2 gene, a non-synonymous mutation, which is an AGC to ATC variant resulting in an S323 to I substitution. This led to altered ASP bioactivity and was associated with FCH in a French-Canada family. Nevertheless, the distribution of this variant in other ethnic groups remains unclear. 


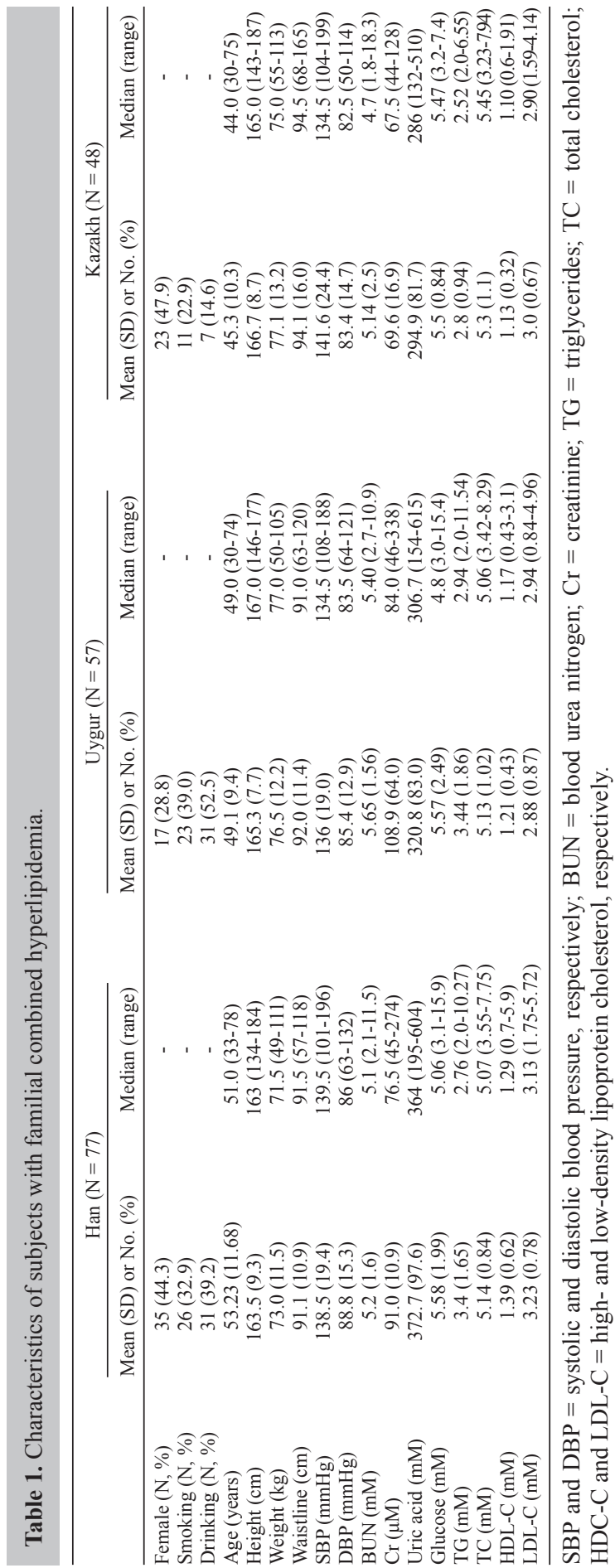




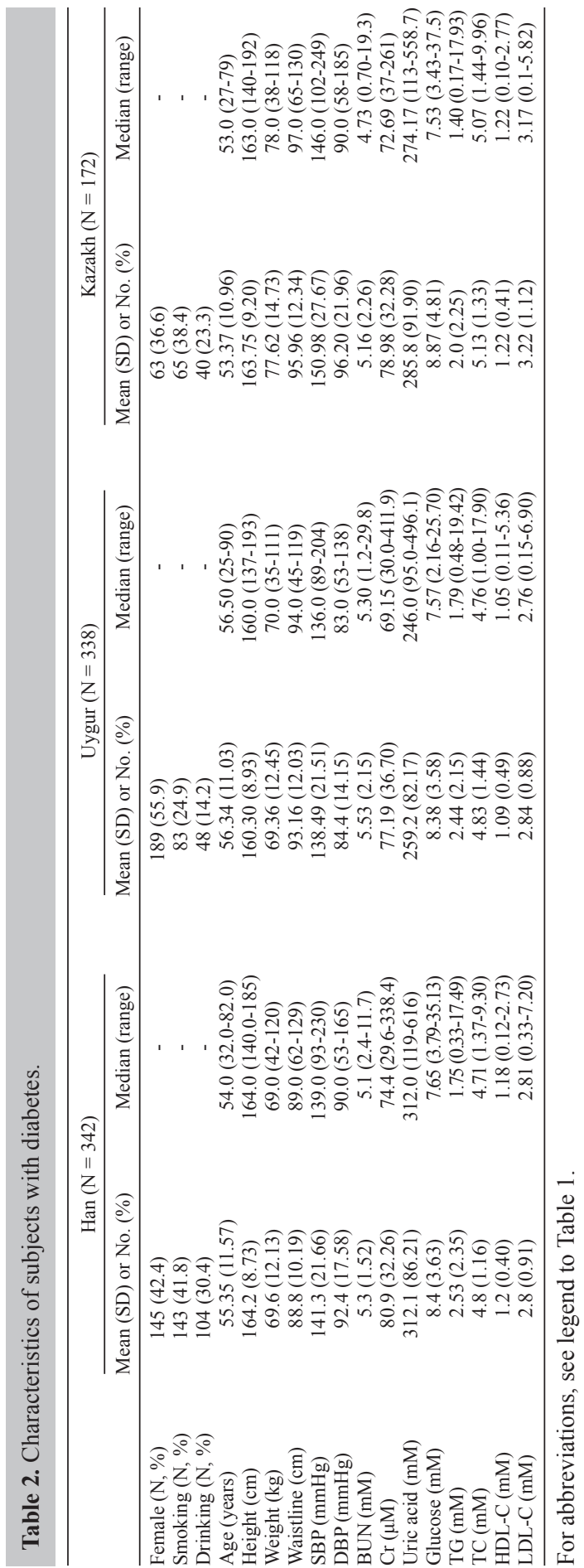




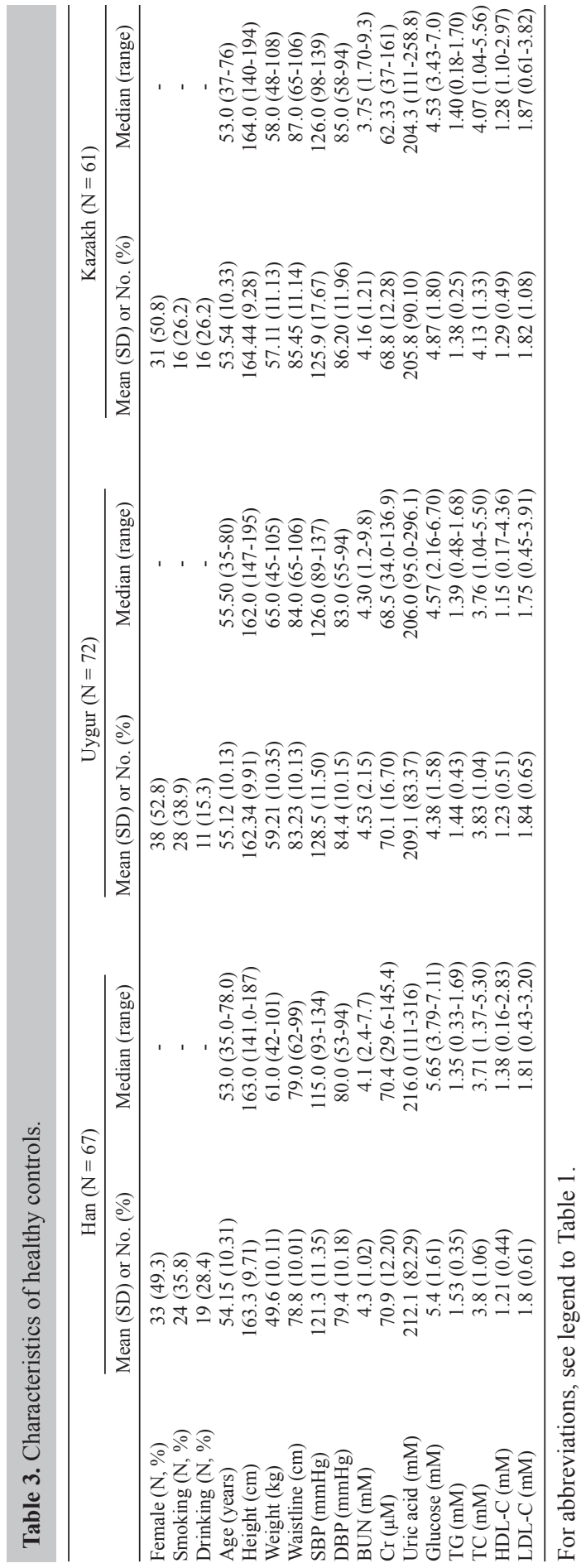


Familial combined hyperglycemia, DM and C5L2

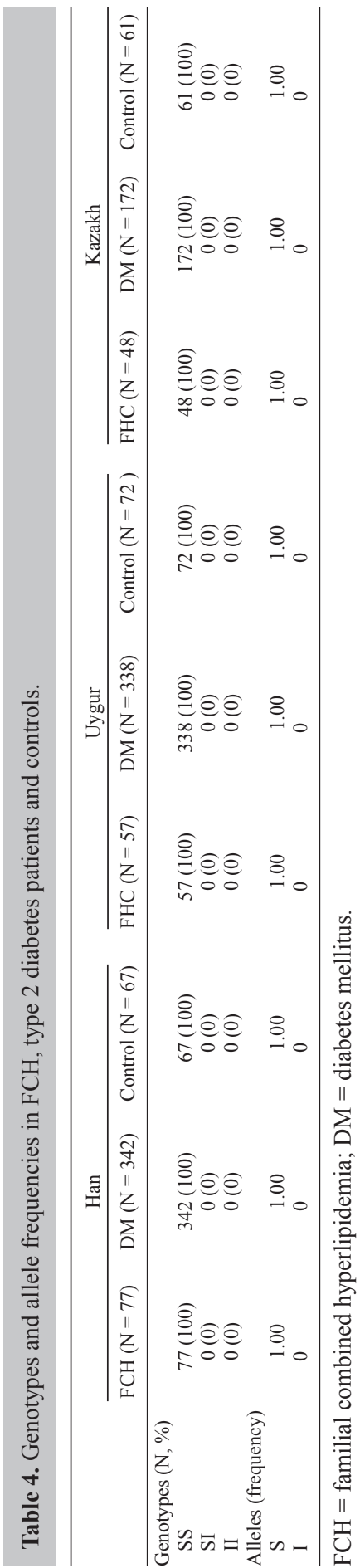


A

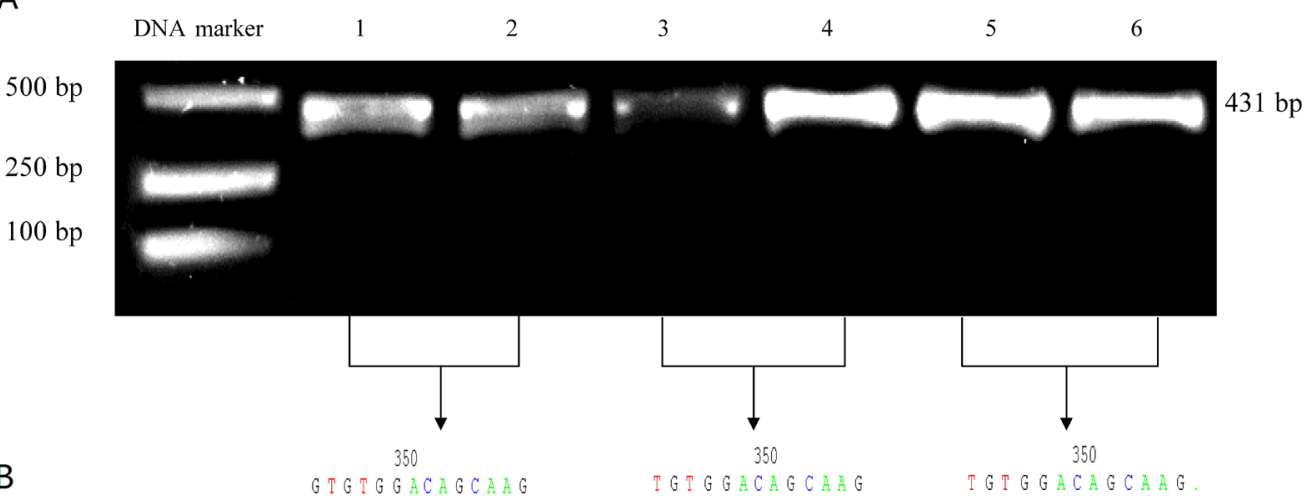

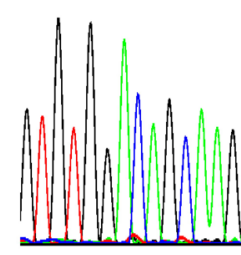

Han

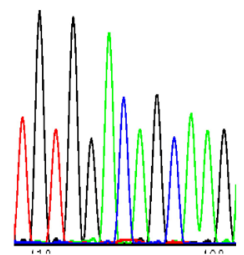

Uygur

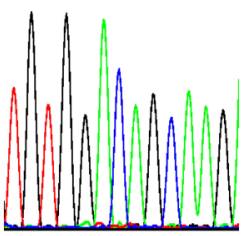

Kazakh

Figure 1. A. Restriction fragment length polymorphism analysis for determination of genotype. The SS genotype shows a single band of 431 bp (lanes 1 and 2 indicate the Han ethnic group; lanes 3 and 4 indicate the Uygur ethnic group; lanes 5 and 6 indicate the Kazakh ethnic group). B. Nucleotide sequences around S323I polymorphism in exon 2 .

China is the most populous country in the world and with 56 ethnic groups. The Han ethnic group makes up over $92 \%$ of China's population. The remaining 55 ethnic groups are collectively called minorities. Xinjiang is part of the ancient Silk Road and borders eight countries including Russia, Kazakhstan, Kirghizastan, Tajikistan, Pakistan, Mongolia, India, and Afghanistan. There are more than 13 ethnic groups living in this area. Among them, the Uygur people account for $46 \%$, Han account for $40 \%$, and Kazakh account for $7 \%$. In the present study, we genotyped the S323I variant in these three ethnic groups. It is important to understand the genetic profiles of the C5L2 gene and to recognize the role of S323I variant on FCH and type 2 diabetes in Xinjiang of China. Nevertheless, we did not find the S323I mutation in these three ethnic groups, which may indicate that there was no evidence to demonstrate that the S323I polymorphism is one molecular basis contributing to $\mathrm{FCH}$ and diabetes in the Chinese population. This is a possible explanation for the following reasons: 1) $\mathrm{FCH} /$ diabetes is a multifactorial disorder, and not all subjects have a C5L2 mutation; 2) this mutation (S323I) appears to be relatively rare. Also, there may be other as not yet recognized mutations in the coding region; 3) genotypic milieu may play an important role. Therefore, due to the important role of S323I variant, the distribution of this mutation in other ethnic populations merits further investigation. 


\section{Limitation of this study}

This study has several limitations. First, in the present study we only investigated one variant, S323I, rather than other variants in the C5L2 gene. This fact may underestimate the role of the C5L2 gene although the main aim of the present study was to investigate the role of S323I. Second, due to the absence of additional samples of plasma and serum of the participants, we did not measure the plasma concentrations of ASP and apolipoprotein B. Third, we investigated this variant in three ethnic groups including Han, Uygur, and Kazahk, the remaining 10 minorities in Xinjiang were not investigated in the present study.

\section{CONCLUSIONS}

In these 182 patients with $\mathrm{FCH}$ and 852 subjects with type 2 diabetes, we were not able to identify the S323I variant of the C5L2 gene. This result suggests that the S323I variant may not be a molecular basis contributing to $\mathrm{FCH}$ and type 2 diabetes in the Chinese population.

\section{ACKNOWLEDGMENTS}

Research supported by the Open Project of Xinjiang Key Laboratory of Cardiovascular Disease Research (\#XJDX0903-2009-01).

\section{Conflicts of interesting}

None.

\section{REFERENCES}

Aouizerat BE, Allayee H, Cantor RM, Davis RC, et al. (1999). A genome scan for familial combined hyperlipidemia reveals evidence of linkage with a locus on chromosome 11. Am. J. Hum. Genet. 65: 397-412.

Cianflone K (2003). Acylation stimulating protein and triacylglycerol synthesis: potential drug targets? Curr. Pharm. Des. 9: 1397-1410.

Cianflone K, Xia Z and Chen LY (2003). Critical review of acylation-stimulating protein physiology in humans and rodents. Biochim. Biophys. Acta 1609: 127-143.

Cui W, Lapointe M, Gauvreau D, Kalant D, et al. (2009a). Recombinant C3adesArg/acylation stimulating protein (ASP) is highly bioactive: a critical evaluation of C5L2 binding and 3T3-L1 adipocyte activation. Mol. Immunol. 46: 32073217.

Cui W, Simaan M, Laporte S, Lodge R, et al. (2009b). C5a- and ASP-mediated C5L2 activation, endocytosis and recycling are lost in S323I-C5L2 mutation. Mol. Immunol. 46: 3086-3098.

de Lind van Wijngaarden RF, Cianflone K, Gao Y, Leunissen RW, et al. (2010). Cardiovascular and metabolic risk profile and acylation-stimulating protein levels in children with Prader-Willi syndrome and effects of growth hormone treatment. J. Clin. Endocrinol. Metab. 95: 1758-1766.

Faraj M, Sniderman AD and Cianflone K (2004). ASP enhances in situ lipoprotein lipase activity by increasing fatty acid trapping in adipocytes. J. Lipid Res. 45: 657-666.

Huertas-Vazquez A, Aguilar-Salinas C, Lusis AJ, Cantor RM, et al. (2005). Familial combined hyperlipidemia in Mexicans: association with upstream transcription factor 1 and linkage on chromosome 16q24.1. Arterioscler. Thromb. Vasc. Biol. 25: 1985-1991.

Kalant D, MacLaren R, Cui W, Samanta R, et al. (2005). C5L2 is a functional receptor for acylation-stimulating protein. J. Biol. Chem. 280: 23936-23944.

MacLaren R, Kalant D and Cianflone K (2007). The ASP receptor C5L2 is regulated by metabolic hormones associated 
with insulin resistance. Biochem. Cell Biol. 85: 11-21.

Marcil M, Vu H, Cui W, Dastani Z, et al. (2006). Identification of a novel C5L2 variant (S323I) in a French Canadian family with familial combined hyperlipemia. Arterioscler. Thromb. Vasc. Biol. 26: 1619-1625.

Maslowska M, Wang HW and Cianflone K (2005). Novel roles for acylation stimulating protein/C3adesArg: a review of recent in vitro and in vivo evidence. Vitam. Horm. 70: 309-332.

Paglialunga S, Julien P, Tahiri Y, Cadelis F, et al. (2009). Lipoprotein lipase deficiency is associated with elevated acylation stimulating protein plasma levels. J. Lipid Res. 50: 1109-1119.

Saleh J, Al-Khanbashi M, Al-Maarof M, Al-Lawati M, et al. (2009). Acylation-stimulating protein increases and correlates with increased progesterone levels during the luteal phase of the menstrual cycle. Eur. J. Endocrinol. 160: 301-307.

Xiang SQ, Cianflone K, Kalant D and Sniderman AD (1999). Differential binding of triglyceride-rich lipoproteins to lipoprotein lipase. J. Lipid Res. 40: 1655-1663.

Xiang X, Ma YT, Fu ZY, Yang YN, et al. (2009). Haplotype analysis of the CYP8A1 gene associated with myocardial infarction. Clin. Appl. Thromb. Hemost. 15: 574-580.

Xie X, Ma YT, Fu ZY, Yang YN, et al. (2009). Association of polymorphisms of PTGS2 and CYP8A1 with myocardial infarction. Clin. Chem. Lab. Med. 47: 347-352.

Xie X, Ma YT, Yang YN, Li XM, et al. (2010a). Alcohol consumption and ankle-to-brachial index: results from the Cardiovascular Risk Survey. PLoS One 5: e15181.

Xie X, Ma YT, Yang YN, Fu ZY, et al. (2010b). Polymorphisms in the SAA1/2 gene are associated with carotid intima media thickness in healthy Han Chinese subjects: the Cardiovascular Risk Survey. PLoS One 5: e13997.

Yang YN, Wang XL, Ma YT, Xie X, et al. (2010). Association of interaction between smoking and CYP 2C19*3 polymorphism with coronary artery disease in a Uighur population. Clin. Appl. Thromb. Hemost. 16: 579-583. 